\title{
Wideband RCS reduction of thin metallic edges mediated by spoof surface plasmon polaritons
}

\author{
Xinghua $\mathrm{Li}^{1}$, Mingde Feng ${ }^{1, *}$, Jiafu Wang ${ }^{1, * *}$, Xinmin $\mathrm{Fu}^{1}$, Yajuan $\mathrm{Han}^{1}$, Sai Sui ${ }^{1}$, Yongqiang Pang ${ }^{2}$, \\ and Shaobo $\mathrm{Qu}^{1}$ \\ ${ }^{1}$ Department of Basic Sciences, Air Force Engineering University, Xi'an, Shaanxi 710051, PR China \\ 2 School of Electronics and Information Engineering, Xi'an Jiaotong University, Xi'an, Shaanxi 710049, PR China
}

Received: 17 October 2020 / Accepted: 25 December 2020

\begin{abstract}
The back-scattering from front edge diffraction contributes significantly to mono-static radar cross section under TE-polarization when the specular reflection of an object is eliminated by elaborate shaping. With the aim to suppress the back-scattering of thin metallic edge, we propose to achieve wideband radar cross section (RCS) reduction by integrating an absorbing structure (AS) in front of the edge. The unit cell of AS is composed of a longitudinal array of metallic strips with linearly decreasing lengths. Under TE-polarized illumination, spoof surface plasmon polariton (SSPP) can be excited with high efficiency. Due to the deep-subwavelength property of SSPP, electromagnetic waves are highly confined around the AS, leading to strong local field enhancement and hence to wideband absorption. In this way, back-scattering of the edge is suppressed and the mono-static RCS can be reduced significantly over wide band. To verify this method, we designed, fabricated and measured a prototype. The results of both simulation and measurement indicate that our proposal can significantly suppress edge scattering, whose RCS reduction more than $10 \mathrm{~dB}$ achieves at range of 8.8-17.8 GHz under TE polarization. This work provides a new alternative of suppressing edge diffraction and may find applications in electromagnetic compatibility, radar stealth, etc.
\end{abstract}

Keywords: Edge diffraction / radar cross section reduction / radar absorbing structure / spoof surface plasmon polaritons

\section{Introduction}

The edge diffraction as potential scattering source is existed extensively on discontinuous surfaces or boundaries, which can be interpreted by the impedance mismatch between the object edges and their surroundings. In fact, the radar echoes of edge diffraction is only a minor part compared to that of specular reflection. However, when the specular reflection is effectively controlled by the shape design and radar absorber material or the incident plane wave is in a grazing condition, the edge diffraction becomes the major contributor to radar cross section (RCS). For engineering applications, it is crucial to reduce RCS caused by the edge diffraction that in order to improve the performance of the research targets [1-3].

The edge diffraction can be suppressed by the combination of shaping geometry and designing material property [4,5]. Methods for suppressing edge diffraction include the use of edge serration [6-8], edge corrugations

\footnotetext{
* e-mail: fmingde@mail.xjtu.edu.cn

** e-mail: wangjiafu1981@126.com
}

[9], resistive tapers loading [10-12], and edge coatings $[13,14]$, which have received considerable attention. Other related works have involved using the impedance surface to redirect the back-scattering, orto guide the surface waves toward or away from the hypotenuse $[15,16]$. The focus of methods following the shape design principle is the deflection of edge diffraction, which may deteriorate other engineering indicators, such as weight or aerodynamic performance $[5,15]$. For material property design, resistive tapers loading is an attractive method for suppressing the edge diffraction by providing a gradual transition from a metallic edge to free space, which is of a remarkable interest for many stealth engineers [17]. However, it also suffers from an obvious limitation of narrow bandwidth.In fact, the wideband suppression of edge diffraction is not only highly demanded but also challenged for many engineering applications. Note that spoof surface plasmon polariton (SSPP) is serving for wideband absorption by virtue of deep-subwavelength and strong local field enhancement properties [18]. The wideband absorption of periodically arranged SSPP framework has been introduced in several recent reference papers [19-24]. In this work, we expand on 


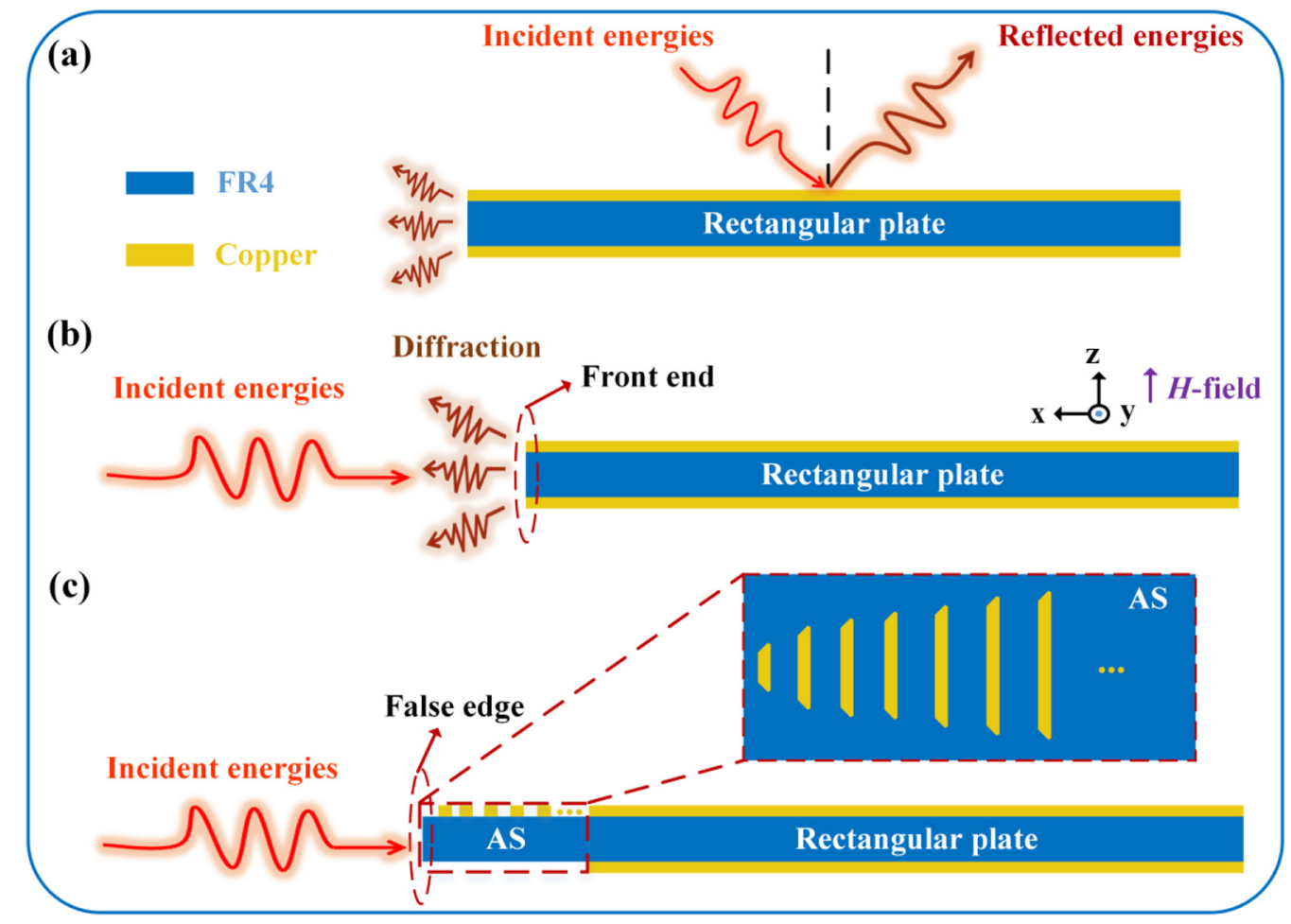

Fig. 1. Edge diffraction suppression design. (a) The thin metallic front edges of a rectangular plate scatter the TE polarized wave at an oblique incident angle. (b) The edge diffraction is the major scattering source under the horizontal incidence. (c) An absorbing structure (AS) is adopted to suppress the edge diffraction.

the previous publications to integrate a specific application scene, where an absorbing structure (AS) based on SSPP [24] is applied to realize the aim of wideband suppression of edge diffraction.

The design principle of this paper is illustrated in Figure 1: under the TE polarization condition, the diffraction deriving from the thin metallic front edges is induced along with the specular reflection when the plane wave is obliquely incident on a rectangular plate. The term TE polarization means the electric field of the plane wave is perpendicular to the incident plane ( $x-z$ plane). Considering the distance between the source and objects, the incident plane wave is often in a grazing condition when it comes to detected objects [25]. In this case, the diffraction from the front edges is a major scattering source [26]. In order to reduce mono-static RCS caused by the edge diffraction under the TE polarization, the design guidance is to use the AS to suppress the edge backscattering. Different from the traditional absorbing material, this work suggests a new strategy which involves replacing the thin metallic front edges of rectangular plate by the SSPP framework of AS. This would result in highly effective excitation of SSPP and hence a great elimination of the edge diffraction in the backward direction. A series of procedures of simulations and experiments are carried out to verify the back-scattering suppression performance of our proposal. As we expected, both results indicate that this approach is highly effective at the issues of suppressing edge diffraction in wideband frequency range.

\section{Scattering property of a rectangular plate}

When the plane wave illuminates a rectangular plate with thin metallic edges under the TE polarization, the edge diffraction can arise on the front edges and hence lead to the increase of RCS. The rectangular plate is used because it has a simple scattering pattern with a single main lobe in the backward direction [15]. For the TE polarization, the electric field $E$ is parallel to the front edges, which drives the free electrons of metallic edges to create the edge current to scatter the diffraction energy [27]. The backscattering of edge diffraction significantly contributes to mono-static RCS in the backward direction, especially when the incident plane wave is in a grazing condition. To verify this, commercial simulation software CST [28] is implemented in discussion for analyzing the intensity of edge current and mono-static RCS of the rectangular plate. In this case, the rectangular plate composed of FR 4 substrate $\left(\varepsilon_{r}=4.3, \tan \delta=0.025\right)$ covered with thin copper plate on both sides is considered for its front edge length $l=240 \mathrm{~mm}$. Considering the practical condition of large angle incidence, the horizontal incidence is taken as the simulation case. The horizontal EM wave is incident in the $-x$ direction and the electric field of the incidence is along to the $y$ axis. The distribution of surface current and the mono-static RCS of the rectangular plate are shown in Figure 2. It is obvious that the current of front edge is more intense than that of other areas, which demonstrates that the edge diffraction results from the edge current. What's more, the surface wave propagates forward along the both 
side edges but has few effect on the backward edge diffraction [2]. Hence, it can be concluded that the front edge diffraction is major scattering source which is crucial to be suppressed. The value of mono-static RCS caused by the edge diffraction is large enough, even for the small rectangular plate. In order to reduce mono-static RCS of thin metallic edges, the AS is applied to the specific scene, where the SSPP framework can only be arranged in a single direction to replaced the thin metallic front edges of the

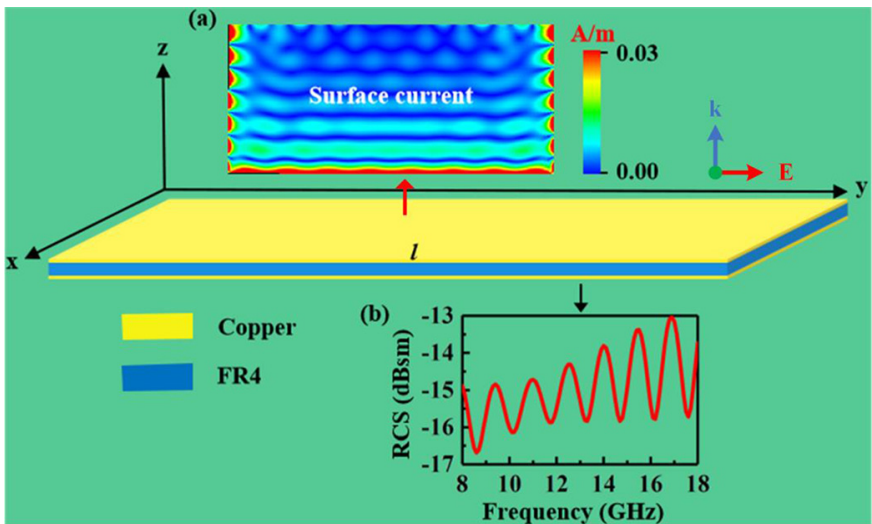

Fig. 2. Schematic of a rectangular plate for its front edge length $l=240 \mathrm{~mm}$. (a) The surface current distribution of the rectangular plate. (b) Mono-static RCS of the rectangular plate. rectangular plate. The configuration of the AS has good absorption performance, which is the foundation of whole design process for the RCS reduction of edge diffraction.

\section{Absorbing structure design and simulation}

The AS is created by several metallic strips with linearly decreasing lengths etched on dielectric substrate with a metal ground as shown in Figure $3 \mathrm{~b}$. At microwave frequencies, the framework of AS have been demonstrated to support the highly effective excitation of SSPP and hence to absorb the electromagnetic wave in wideband frequency by virtue of the deep-subwavelength and strong local field enhancement properties of SSPP. Here we employed such strips to construct the desired absorbing framework. The metal used in the model is copper with the electric conductivity of $5.8 \times 10^{7} \mathrm{~S} / \mathrm{m}$, while the relative permittivity and the loss tangent of the dielectric substrate are 4.3 and 0.025. According to reference [28], the absorption peak is related to the length of metallic strips and is nearly equals to the asymptotic frequency plotted in the dispersion curve of a configuration consisting of several metallic strips of the same length $r$ as shown in Figure 3a. With the increased length $r$ from 4.7 to $10 \mathrm{~mm}$, the absorption peak decreased from 18.5 to $8.8 \mathrm{GHz}$. These metallic strips with different lengths are integrated into the AS, which guaranteed the wideband absorption of the framework. The bottom strip of AS is selected to be $10 \mathrm{~mm}$, and in order to make the impedance match well between

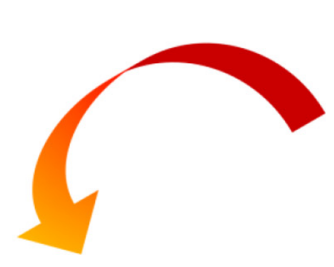

(a)
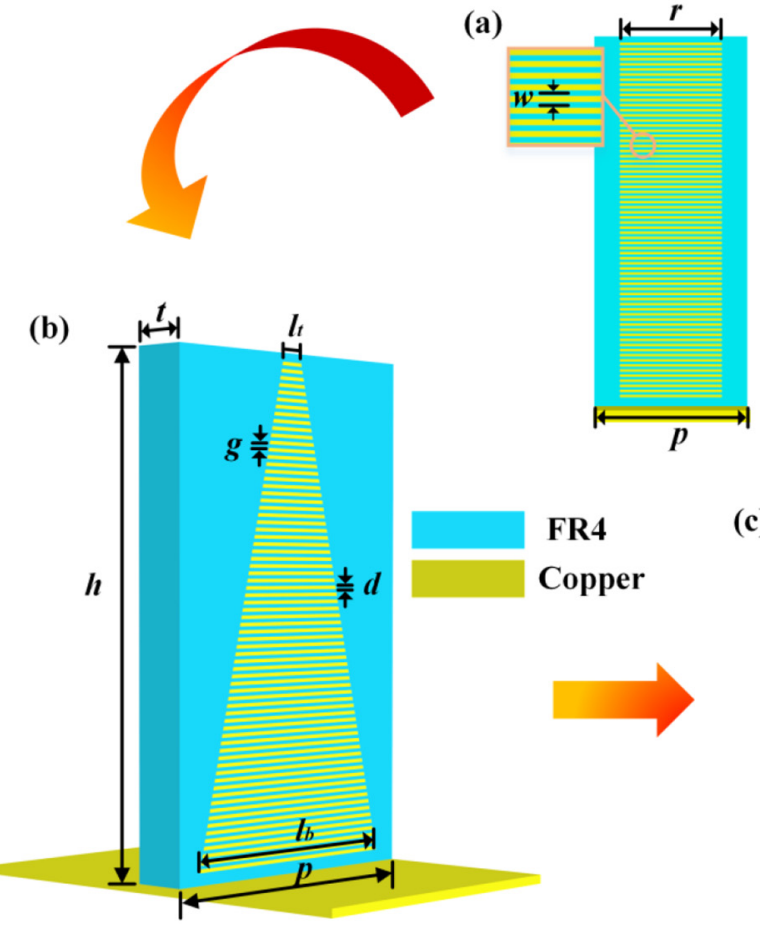

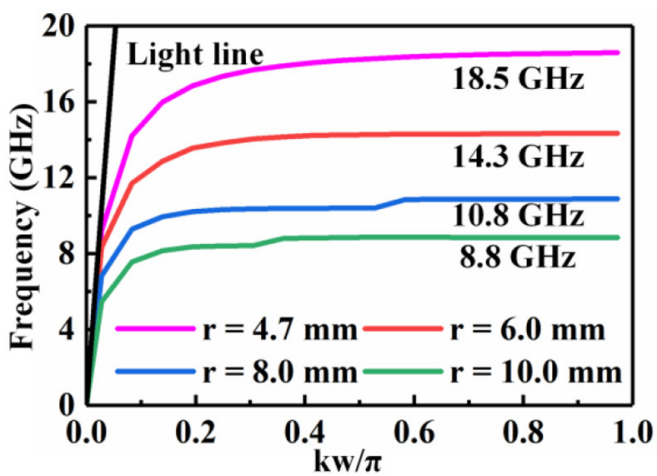

(c)

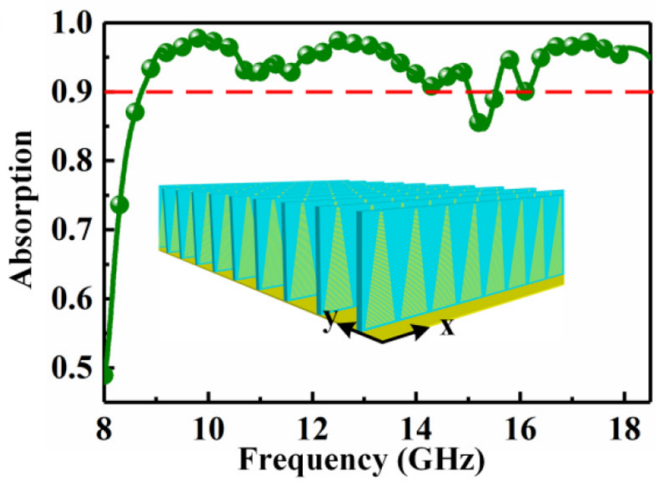

Fig. 3. (a) Calculated dispersion of a configuration consisting of several metallic strips of the same length $r$. (b) Schematic of the absorbing structure (AS). Dimension parameters: $p=12.0 \mathrm{~mm}, h=29.0 \mathrm{~mm}, l_{t}=1.0 \mathrm{~mm}, l_{b}=10.0 \mathrm{~mm}, d=0.2 \mathrm{~mm}, g=0.2 \mathrm{~mm}$, $t=2.0 \mathrm{~mm}$. (c) Absorption spectra of the AS. 

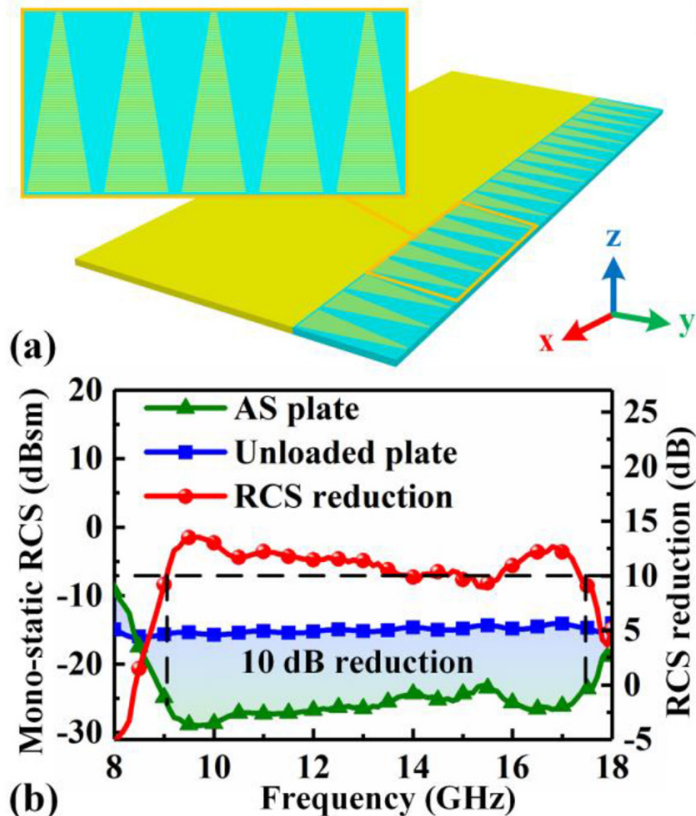

(c)
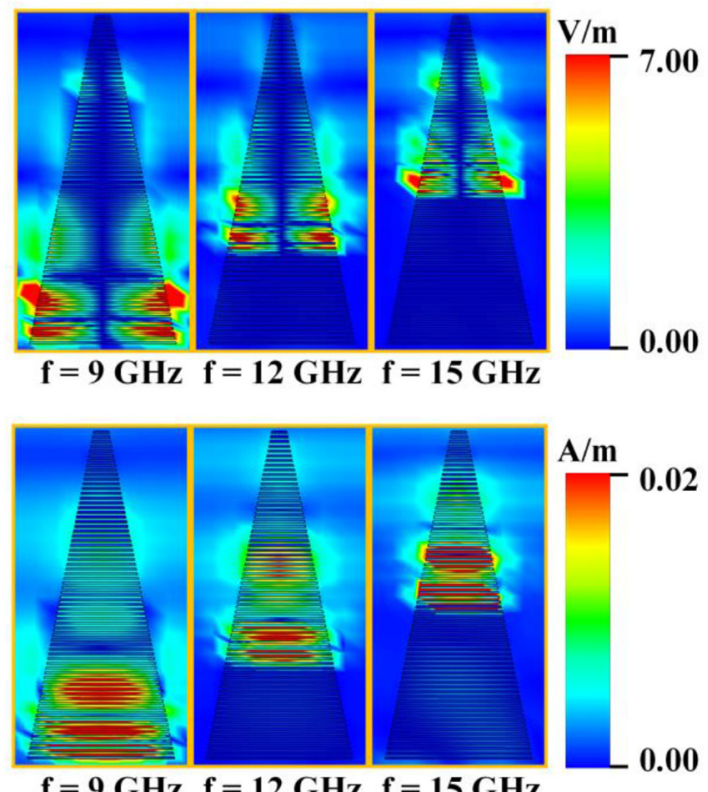

Fig. 4. (a) Schematic of the AS plate. (b) The mono-static RCS of rectangular plate loaded with and without AS. (c) Simulated electric and magnetic field distribution of a AS unit cell at 9, 12 and $15 \mathrm{GHz}$ respectively.

the AS and its surroundings, the length of top strip is decreased to $1 \mathrm{~mm}$. Due to the existence of the metal backplane, the absorption $A(\omega)$ can be characterized by $A(\omega)=1-R(\omega)$, where $R(\omega)$ is the reflectance and has the form of $R(\omega)=\left|S_{11}\right|^{2}$ [28]. Numerical simulation is performed by using the Frequency Domain solver in the CST 2018, and the unit cell boundary condition is employed along $\mathrm{x}$-axis and $\mathrm{y}$-axis directions. The simulation result is shown in Figure 3c, where the AS actually possess a good absorption performance with $\mathrm{A}(\omega)$ above $90 \%$ in $8.8-18.5 \mathrm{GHz}$. It should be noted that there are two points need to be discussed. One is that the good absorption is obtained in a common scene where the AS can be arranged in an infinite period along two directions. But in the application scene studied in this work, the configuration of AS can only be finite period along the single direction, which may degrade the absorption performance over wideband frequency range. The other one is that the framework has many distinct advantages, among which the obvious properties are thin, light and easy implementation. In addition, besides the absorption, a good impedance match can be achieved between the framework and the surrounding due to the decreased filling ratio of the top metallic strips, which avoids the extra scattering caused by the framework itself.

\section{Scattering simulations and measurement}

In the scene of edge diffraction, the metallic front edges of a rectangular plate are major scattering source, which need to be disposed. A direct way is to replace the front edges using the AS. Based on this, the configuration is finite periodic arranged in front of the metallic edges along the $x$ axis direction shown in Figure 4a. The complete plate (AS plate) is located in the $x$ - $y$ plane, with the same dimension as the rectangular plate unloaded with the AS. Suppression performance of the configuration is numerical calculated in the open (add space) boundaries using Time Domain solver in the CST 2018. The simulation result of mono-static RCS is indicated in Figure 4b. It can be seen that a lower mono-static RCS of AS plate is acquired compared with that of unloaded plate, with the RCS reduction over $10 \mathrm{~dB}$ in $8.8-17.5 \mathrm{GHz}$. Note that the suppression bandwidth in this scene is narrow than the absorbing frequency range just mentioned, which also verifies that the absorption of AS is effected by the finite periodic arrangement.

To further understand the suppression mechanism of the AS plate, the electric and magnetic field distributions at some frequencies are depicted in Figure 4c. It is obvious that the electromagnetic field is confined around the AS unit cells. As the frequency increases, the local field region moves gradually towards the top-side from the bottom correspondingly. Therefore, it can be concluded that the SSPP is excited with high efficiency, and its deepsubwavelength property leads to strong local field enhancement and hence to wideband absorption. In sum, we can primarily confirm that the proposal using the AS to suppress the edge diffraction is feasible. It should be noted that the AS is very good for the TE polarized incidence, but not as efficient for TM polarized incidence. That is because the electric field of the TM polarized incidence is not parallel to the metallic strips of the AS, which can not effectively excite the SSPP on the AS and hence can not have a good performance of absorption. In other words, the AS only works under TE polarization rather than TM polarization. 

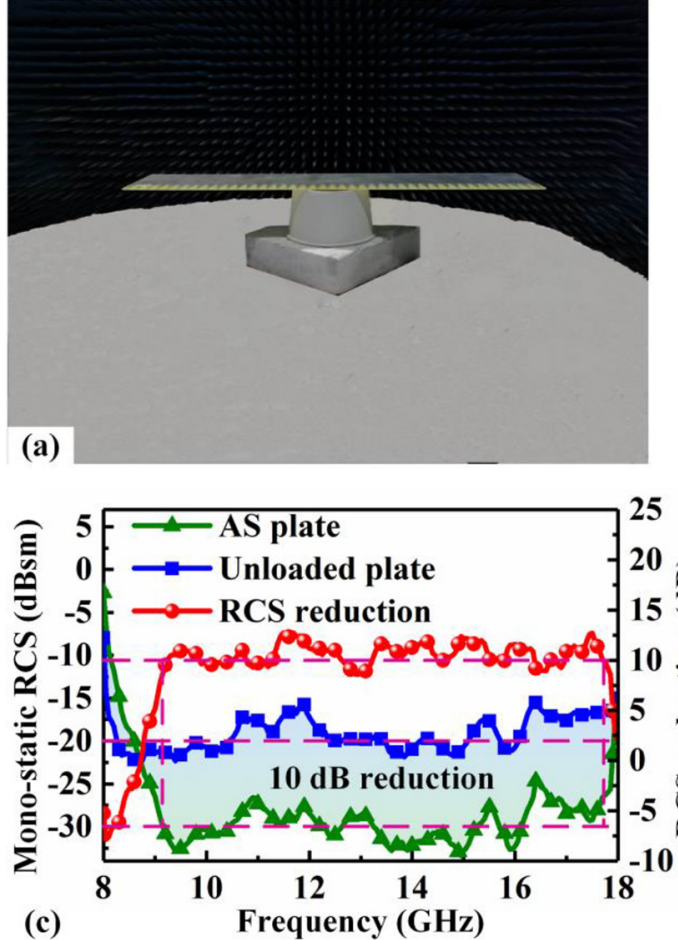

(c)
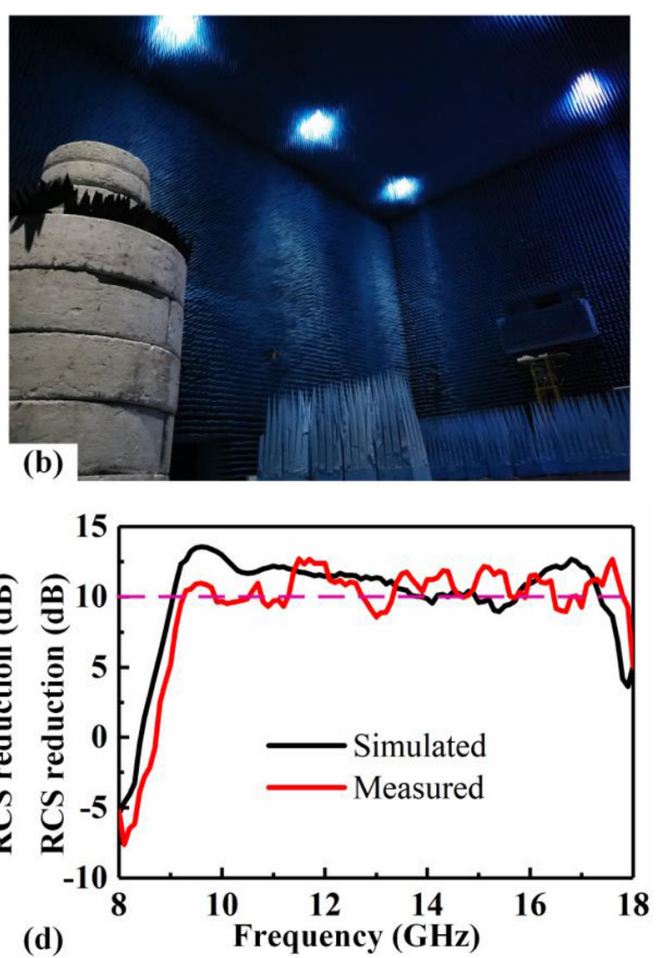

Fig. 5. (a) The photo of the fabricated prototype. (b) Measured environment. (c) The mono-static RCS of rectangular plate loaded with and without AS, and RCS reduction between the two plates. (d) Comparison of simulated and measured RCS reduction.

For further verification, the AS plate with a large dimension is fabricated using Print Circuit Board (PCB) technique. The prototype is $500 \times 90 \mathrm{~mm}$, which has forty AS unit cells fixed in front of the metallic edges. A photograph of the fabricated prototype is shown in Figure 5a. The measurement was performed in a microwave anechoic chamber as shown in Figure 5b. The measured curves of mono-static RCS associated with AS plate and unloaded plate for the same size is presented in Figure 5c. It is obvious that the average value of monostatic RCS of the rectangular plate loaded with AS is lower than that of unloaded one, and a reduction over $10 \mathrm{~dB}$ from 9.0 to $17.8 \mathrm{GHz}$ is obtained, which is roughly in line with the simulated results seen in Figure 5d. Based on this, it can be concluded that the special framework of AS is actually effective at suppressing edge diffraction over wideband frequency range under the TE polarization.

\section{Conclusion}

In summary, a special SSPP framework has been selected to suppress the edge diffraction over wideband frequency range under the TE polarization. In order to reduce the diffraction from the metallic front edges of a rectangular FR4 dielectric plate with thin copper plate on its both sides, an AS unit cell containing several metal strips with their length tapered linearly from the bottom to the top has been then analyzed, which is demonstrated to possess a good absorption performance. With highly efficient excitation of SSPP, the wideband suppression for the edge diffraction has been realized by the AS. Both the simulation and measurement results verified the feasibility of the method using the SSPP framework to suppress edge diffraction. In addition, the design can be incorporated into many applications, such as improving the stealth of aircraft, reducing coupling between the antennas, and improving EM compatibility. What's more, this work only focused on reducing TE polarized scattering, and the incident angle is also limited. So in the next step, we can keep on designing a configuration which is independent on the polarization and the incident angle to suppress the edge diffraction.

The authors are grateful to the supports from the National Natural Science Foundation of China (Grant numbers 61671466, 61971435).

\section{References}

1. J.D. Edmunds, M.C. Taylor, A.P. Hibbins, J.R. Sambles, I.J. Youngs, Babinet's principle and the band structure of surface waves on patterned metal arrays, J. Appl. Phys. 107, 638 (2010)

2. E. Knott, J. Shaeffer, M. Tuley, in Radar cross section (Artech House, Inc., Norwood, MA, 1985), pp. 23-61

3. F. Costa, S. Genovesi, A. Monorchio, A frequency selective absorbing ground plane for low-RCS microstrip antenna arrays. 126, 317 (2012) 
4. J. Kappa, Z. Dang, D. Sokoluk, M. Rahm, Analysis of coding metasurfaces for incident radiation at oblique incidence angles, OSA Contin. 2, 2172 (2019)

5. K. Zikidis, A. Skondras, C. Tokas, Low observable principles, stealth aircraft and anti-stealth technologies, in 2nd Int'l Conf. on Applications of Mathematics and Informatics in Military Sciences (AMIMS), (2014)

6. D. Jenn, Radar and laser cross section engineering (American Institute of Aeronautics and Astronautics, Inc. 2005)

7. W.-H. Choi, B.-S. Kwak, Y.-W. Nam, Radar absorbing serrated edge for broadband radar cross section reduction, Microw. Opt. Technol. Lett. 62, 1112 (2020)

8. L. Zhanhe, H. Peilin, W. Zhe, G. Xu, Analysis of scattering from serrated edge plate on aircraft with MLFMA, J. Beijing Univ. Aeronaut. Astronaut. 34, 499 (2008)

9. W.-S. Lee, S.-J. Lee, D.-J. Lee, W.-S. Lee, J.-W. Yu, TE scattering from concaved wedges with longitudinal corrugations, IEEE Trans. Antennas Propag. 61, 2355 (2013)

10. M. Gustafsson, RCS reduction of integrated antenna arrays with resistive sheets, J. Electromagn. Waves Appl. 20, 27 (2006)

11. M. Gustafsson, Surface integrated dipole arrays with tapered resistive edge sheets, J. Electromagn. Waves Appl. 21, 713 (2007)

12. H.Y. Chen, L.J. Deng, P.H. Zhou, J.L. Xie, Tapered impedance loading for suppression of edge scattering, IET Microw. Antennas Propag. 5, 1744 (2011)

13. F.C. Smith, Edge coatings that reduce monostatic RCS, IEE Proc. Radar Sonar Navig. 149, 310 (2002)

14. Y.W. Nam, J.H. Choi, M.S. Jang, W.J. Lee, C.G. Kim, Radar-absorbing structure with nickel-coated glass fabric and its application to a wing airfoil model, Compos. Struct. 180, 507 (2017)

15. Q. Ryan, D.F. Sievenpiper, Alteration of electromagnetic scattering using hard and soft anisotropic impedance surfaces, IEEE Trans. Antennas Propag. 63, 4593 (2015)

16. H. Hou, J. Long, J. Wang, D.F. Sievenpiper, Reduced electromagnetic edge scattering using inhomogeneous anisotropic impedance surfaces, IEEE Trans. Antennas Propag. 65, 1193 (2017)
17. H.Y. Chen, Z.W. Zhu, L.J. Lu, Y. Guan, J.L. Xie, L.J. Deng, Design and implementation of the tapered resistive sheets to control edge scattering, J. Appl. Phys. 115, 164906 (2014)

18. Y. Pang, J. Wang, H. Ma, M. Feng, Y. Li, Z. Xu et al., Spatial $\mathrm{k}$-dispersion engineering of spoof surface plasmon polaritons for customized absorption, Sci. Rep. 6, 29429 (2016)

19. Y. Shen, J. Zhang, B. Dong, Y. Pang, J. Wang, H. Ma et al., Plasmonic absorbing structure using horizontal bent-wire array for low-frequency absorption enhancement, Opt. Commun. 443, 90 (2019)

20. Y. Han, S. Gong, J. Wang, Y. Li, S. Qu, J. Zhang, Reducing RCS of patch antennas via dispersion engineering of metamaterial absorbers. IEEE Trans. Antennas Propag. 68, 1419 (2020)

21. Y. Fan, J. Wang, X. Fu, Y. Li, Y. Pang, L. Zheng et al., Recent developments of metamaterials/metasurfaces for RCS reduction, EPJ Appl. Metamater. 6, 15 (2019)

22. Y. Han, W. Jiang, J. Wang, S. Gong, Y. Li, L. Zheng, J. Zhang, A. Zhang, S. Qu, Multi-functional sandwich structure with metamaterial antenna lattice cores: protection, radiation and absorption, IET Microw. Antennas Propag. 14, 593 (2020)

23. R. Zhu, J. Wang, S. Sui, Y. Meng, S. Qu, Wideband absorbing plasmonic structures via profile optimization based on genetic algorithm, Front. Phys. 8, 231 (2020)

24. F. Ding, Y. Cui, X. Ge, Y. Jin, S. He, Ultra-broadband microwave metamaterial absorber, Appl. Phys. Lett. 100, $103506(2012)$

25. Y. Zhou, X.-Y. Cao, J. Gao, S. Li, X. Liu, RCS reduction for grazing incidence based on coding metasurface, Electron. Lett. 53, 1381 (2017)

26. P.Y. Ufifimtsev, Method of edge waves in the physical theory of diffraction (U.S. Air Force, Foreign Technology Division, Dayton, OH, 1971)

27. C. Haiyan, X. Jianliang, Z. Zhiwei, D. Longjiang, Method of tapered resistive sheet loading for controlling edge scattering, Microw. Opt. Technol. Lett. 55, 1992 (2013)

28. Z. Wang, X. Fu, J. Wang, Y. Fan, S. Qu, Multi-octave RCS reduction via integrated dispersion engineering of polarization-conversion metasurface and metamaterial absorber, J. Phys. D Appl. Phys. 3, 53 (2019)

Cite this article as: Xinghua Li, Mingde Feng, Jiafu Wang, Xinmin Fu, Yajuan Han, Sai Sui, Yongqiang Pang, Shaobo Qu, Wideband RCS reduction of thin metallic edges mediated by spoof surface plasmon polaritons, EPJ Appl. Metamat. 8, 8 (2021) 